\title{
Einstein-Klein-Gordon System in Higher Dimensional
}

\author{
Mirda Prisma Wijayanto ${ }^{1 *}$, Fiki Taufik Akbar Sobar ${ }^{1,2}$, Bobby Eka Gunara ${ }^{1,2}$ \\ ${ }^{1}$ Theoretical of High Energy Physics Research Division, Institut Teknologi Bandung \\ ${ }^{2}$ Indonesia Center for Theoretical and Mathematical Physics (ICTMP)
}

( Received: December 23, 2021, Revised: December 29, 2021, Accepted: December 29, 2021)

\begin{abstract}
In this present work, we study the Einstein equation coupled with the nonlinear Klein-Gordon equation. We obtain Ricci tensor, scalar curvature, and Einstein equation of the Einstein-Klein-Gordon system in higher dimensional. If we put $D=$ 4, our formulations reduce to the four dimensional Einstein-Klein-Gordon system.
\end{abstract}

Keywords: Einstein equation, Klein-Gordon equation

\section{INTRODUCTION}

The Cauchy problem for Einstein equation was first started by Choquet-Bruhat [4] and the local existence of solutions was proved by using the harmonic coordinates $[7,9,13]$. One of the most remarkable discoveries was the global existence of the Einstein vacuum equation by Christodoulou and Kleinermen [6]. Furthermore, the global existence of Einstein equation in closed space-time has also been proven by Andersson and Moncrief [2]. Then, the Einstein-Maxwell-Yang-Mills equation for small initial data has been studied by Friedrich [8].

It is also interesting to study global properties of solutions to various Einstein-matter equations. Global existence with small data for Einstein equation coupled with other materials such as selfgravitating scalar system has been done by Christodoulou [5], and self-gravitating VlasovPoisson system has been done by Rein and Rendall [14], both of which assume that space-time is spherically symmetric.

Now, let us consider Einstein equation coupled with nonlinear Klein-Gordon equation. In general relativity, this system provides an important toy model for the study of gravitational collapse which retains relevant dynamical degrees of freedom under the assumption of spherical symmetry. Study of Klein-Gordon equation in the Minkowski space frame has been extensively studied, including by Struwe $[1,10,11,15,16]$ and the references therein. The study about nonlinear Klein-Gordon equations coupled with Einstein equations in spherical symmetry has been done previously by Malec [12], where Cauchy data is given on spacelike hypersurface, as well as by examining the local existence of the Cauchy problem outside outgoing null hypersurfaces. Moreover, Chae [3] studied the characteristics of the initial value problem of Einstein equation coupled with the nonlinear Klein-Gordon equation on the four dimension. Hereafter, this system is referred to Einstein-Klein-Gordon (EKG) system. In this present works, we study EinsteinKlein-Gordon system in higher dimensional.

\section{FOUR DIMENSIONAL EKG}

Let us consider Lorentzian manifold diffeomorphic to $R^{4}$. The group $S O(3)$ acts as an isometry and the group orbits are the metric spacelike 2 -spheres in which the invariants of the group form a timelike curve in the spacetime. Then, we introduce the function

\footnotetext{
* Corresponding author.

E-mail address: mirda.prisma.wijayanto@students.itb.ac.id
} 


$$
r=\sqrt{\frac{A}{4 \pi}}
$$

where $A$ is the area of the 2 -sphere. The metric on the 2 -sphere is given by [3]

$$
d s^{2}=r^{2} d \Sigma^{2}=r^{2}\left(d \theta^{2}+\sin ^{2} \theta d \Phi^{2}\right) .
$$

We define a new coordinate $u$ such that the metric (2) can be written as

$$
d s^{2}=-e^{2 F} d u^{2}-2 e^{F+G} d u d r+r^{2} d \Sigma^{2}
$$

where the function $F$ dan $G$ tend to 0 as $r$ goes to infinity according to asymptotic flatness. Metric (3) has non-zero components as follows

$$
\begin{aligned}
& g_{00}=-e^{2 F} \\
& g_{01}=g_{10}=-e^{F+G} \\
& g_{22}=r^{2} \\
& g_{33}=r^{2} \sin ^{2} \theta
\end{aligned}
$$

with the inverse components

$$
\begin{aligned}
& g^{01}=g^{10}=-e^{-(F-G)} \\
& g^{11}=e^{-2 G} \\
& g^{22}=\frac{1}{r^{2}} \\
& g^{33}=\frac{1}{r^{2} \sin ^{2} \theta} .
\end{aligned}
$$

We obtain the non-zero Christoffel symbols of metric (3) as follows

$$
\begin{aligned}
& \Gamma_{00}^{0}=\partial_{0}(F+G)-e^{F-G} \partial_{1} F \\
& \Gamma_{22}^{0}=r e^{-(F+G)} \\
& \Gamma_{33}^{0}=r e^{-(F+G)} \sin ^{2} \theta \\
& \Gamma_{00}^{1}=-e^{F-G} \partial_{0} G+e^{2(F-G)} \partial_{1} F \\
& \Gamma_{01}^{1}=\Gamma_{10}^{1}=e^{F-G} \partial_{1} F \\
& \Gamma_{11}^{1}=\partial_{1}(F+G) \\
& \Gamma_{22}^{1}=-r e^{-(F+G)} \\
& \Gamma_{33}^{1}=-r e^{-2 G} \sin ^{2} \theta \\
& \Gamma_{12}^{2}=\Gamma_{21}^{2}=\Gamma_{13}^{3}=\Gamma_{31}^{3}=\frac{1}{r} \\
& \Gamma_{33}^{2}=-\sin \theta \cos \theta \\
& \Gamma_{23}^{3}=\Gamma_{32}^{3}=\cot \theta
\end{aligned}
$$

From the above Christoffel symbols, we obtain Ricci tensor as follows

$$
\begin{aligned}
R_{00}= & -e^{F-G}\left[\partial_{0} \partial_{1}(F+G)+\frac{2}{r} \partial_{0} G\right] \\
& +e^{2(F-G)}\left[\left(\partial_{1} F\right)^{2}+\partial_{1}\left(\partial_{1} F\right)-\right. \\
& \left.\partial_{1} F \partial_{1} G+\frac{2}{r} \partial_{1} F\right] \\
R_{01}= & R_{10}=-\partial_{0} \partial_{1}(F+G) \\
& +e^{F-G} \partial_{1}(F-G) \partial_{1} F+ \\
& e^{F-G} \partial_{1}\left(\partial_{1} F\right)+\frac{2}{r} e^{F-G} \partial_{1} F \\
R_{11}= & \frac{2}{r} \partial_{1}(F+G)
\end{aligned}
$$

$$
\begin{aligned}
& R_{22}=1-e^{-2 G}\left(r \partial_{1}(F-G)+1\right) \\
& R_{33}=\sin ^{2} \theta\left[1-e^{-2 G}\left(r \partial_{1}(F-G)+1\right)\right]
\end{aligned}
$$

Furthermore, we obtain the scalar curvature

$$
\begin{aligned}
R= & \frac{2}{r^{2}}+2 e^{-(F+G)} \partial_{0} \partial_{1}(F+G) \\
& +e^{-2 G}\left[-2\left(\partial_{1} F\right)^{2}-2 \partial_{1}\left(\partial_{1} F\right)\right. \\
& \left.+2\left(\partial_{1} F\right)\left(\partial_{1} G\right)-\frac{4}{r} \partial_{1}(F-G)-\frac{2}{r^{2}}\right] .
\end{aligned}
$$

The Einstein equation coupled with the nonlinear Klein-Gordon equation fullfiles

$$
R_{\mu \nu}=8 \pi\left(T_{\mu \nu}-\frac{1}{2} g_{\mu \nu} T\right),
$$

with the energy-momentum tensor

$$
\begin{aligned}
T_{\mu \nu}= & \partial_{\mu} \phi \partial_{\nu} \phi-\frac{1}{2} g_{\mu \nu} g^{\alpha \beta} \partial_{\alpha} \phi \partial_{\beta} \phi \\
& -g_{\mu \nu} V(\phi),
\end{aligned}
$$

the scalar potential $V(\phi)$, and $T$ is the trace of $T_{\mu \nu}$. Thus, from the matter field equation

$$
\nabla_{\mu} T^{\mu \nu}=0
$$

we obtain nonlinear Klein-Gordon equation

$$
\square \phi=\frac{\partial V(\phi)}{\partial \phi}
$$

where $\square$ is defined as the d'Alembert operator as follows

$$
\begin{aligned}
& \square \phi=-2 e^{-F-G}\left(\frac{\partial^{2} \phi}{\partial u \partial r}+\frac{1}{r} \frac{\partial \phi}{\partial u}\right) \\
& +e^{-2 G}\left[\frac{\partial^{2} \phi}{\partial r^{2}}+\left(\frac{2}{r}+\frac{\partial}{\partial r}(F-G)\right) \frac{\partial \phi}{\partial r}\right] .
\end{aligned}
$$

The $\{r r\}$ component of (29) can be written down as

$$
\frac{\partial}{\partial r}(F+G)=4 \pi r\left(\frac{\partial \phi}{\partial r}\right)^{2} \text {. }
$$

The $\{\theta \theta\}$ or $\{\Phi \Phi\}$ component of (29) can be written down as

$\frac{\partial}{\partial r}(F-G)+\frac{1}{r}\left(e^{2 G}-1\right)=-8 \pi r e^{2 G} V(\phi)$.

\section{HIGHER DIMENSIONAL EKG}

In higher dimensional, we can written down the metric (3) as

$d s^{2}=-e^{2 F} d u^{2}-2 e^{F+G} d u d r+r^{2} d \Sigma^{d-2}$

with the non-zero metric components

$$
\begin{aligned}
& g_{00}=-e^{2 F} \\
& g_{01}=g_{10}=-e^{F+G} \\
& g_{i j}=r^{2} \hat{\sigma}_{i j}
\end{aligned}
$$

where $\hat{\sigma}_{22}=1$ and $\hat{\sigma}_{33}=\sin ^{2} \theta$. Then, we have the inverse of the metric components

$$
\begin{aligned}
& g^{01}=g^{10}=-e^{-(F-G)} \\
& g^{11}=e^{-2 G} \\
& g^{i j}=\frac{1}{r^{2}} \hat{\sigma}^{i j}
\end{aligned}
$$


where $\hat{\sigma}^{22}=1$ and $\hat{\sigma}^{33}=\sin ^{2} \theta$. We obtain the nonzero Christoffel symbols of metric (36) as follows

$$
\begin{aligned}
& \Gamma_{00}^{0}=\partial_{0}(F+G)-e^{F-G} \partial_{1} F \\
& \Gamma_{i j}^{0}=r e^{-(F+G)} \hat{\sigma}_{i j} \\
& \Gamma_{00}^{1}=-e^{F-G} \partial_{0} G+e^{2(F-G)} \partial_{1} F \\
& \Gamma_{01}^{1}=\Gamma_{10}^{1}=e^{F-G} \partial_{1} F \\
& \Gamma_{11}^{1}=\partial_{1}(F+G) \\
& \Gamma_{i j}^{1}=-r e^{-2 G} \hat{\sigma}_{i j} \\
& \Gamma_{1 j}^{i}=\Gamma_{j 1}^{i}=\frac{1}{r} \delta_{j}^{i} \\
& \Gamma_{i j}^{k}=\hat{\Gamma}_{i j}^{k}(\hat{\sigma})
\end{aligned}
$$

From the above Christoffel symbols, we obtain Ricci tensor as follows

$$
\begin{aligned}
R_{00}= & -e^{F-G}\left[\partial_{0} \partial_{1}(F+G)+\frac{(D-2)}{r} \partial_{0} G\right] \\
& +e^{2(F-G)}\left[\left(\partial_{1} F\right)^{2}+\partial_{1}\left(\partial_{1} F\right)\right. \\
& \left.-\partial_{1} F \partial_{1} G+\frac{(D-2)}{r} \partial_{1} F\right] \\
R_{01}= & R_{10}=-\partial_{0} \partial_{1}(F+G) \\
& +e^{F-G} \partial_{1}(F-G) \partial_{1} F+ \\
& e^{F-G} \partial_{1}\left(\partial_{1} F\right)+\frac{(D-2)}{r} e^{F-G} \partial_{1} F \\
R_{11}= & \frac{(D-2)}{r} \partial_{1}(F+G) \\
R_{i j}= & \hat{R}_{i j}(\hat{\sigma})-e^{-2 G}\left[r \partial_{1}(F-G)\right. \\
& +D-3] \hat{\sigma}_{i j}
\end{aligned}
$$

with

$$
\hat{R}_{i j}(\hat{\sigma})=\partial_{k} \Gamma_{i j}^{k}-\partial_{j} \Gamma_{i k}^{k}+\Gamma_{i j}^{k} \Gamma_{k \lambda}^{\lambda}-\Gamma_{i \lambda}^{k} \Gamma_{k j}^{\lambda}
$$

where

$$
\begin{gathered}
\hat{R}_{22}=1 \\
\hat{R}_{33}=\sin ^{2} \theta
\end{gathered}
$$

If we put $D=4$, equation $(51-54)$ can be reduced to $(23-27)$.

Thus, the scalar curvature can be written down as

$$
\begin{aligned}
R= & \frac{1}{r^{2}} \hat{R}(\hat{\sigma})+2 e^{-(F+G)} \partial_{0} \partial_{1}(F+G) \\
& +e^{-2 G}\left[-2\left(\partial_{1} F\right)^{2}-2 \partial_{1}\left(\partial_{1} F\right)\right. \\
& +2\left(\partial_{1} F\right)\left(\partial_{1} G\right)-\frac{2(D-2)}{r} \partial_{1}(F-G) \\
& \left.-\frac{(D-2)(D-3)}{r^{2}}\right],
\end{aligned}
$$

with

$$
\hat{R}(\hat{\sigma})=\hat{\sigma}^{i j} \hat{R}_{i j}(\hat{\sigma}) .
$$

In four dimensional spherical coordinates, we have $\hat{R}(\hat{\sigma})=2$. Hence, if we put $D=4$, equation (59) can be reduced to (28).
The Einstein equation coupled with the nonlinear Klein-Gordon equation in higher dimensional fullfiles

$$
R_{\mu \nu}=8 \pi\left(T_{\mu \nu}-\frac{1}{(D-2)} g_{\mu \nu} T\right)
$$

We obtain nonlinear Klein-Gordon equation correspond to (38) in higher dimensional, with d'Alembert operator as follows

$$
\begin{aligned}
& \square \phi=-2 e^{-F-G}\left(\frac{\partial^{2} \phi}{\partial u \partial r}+\frac{(D-2)}{2 r} \frac{\partial \phi}{\partial u}\right) \\
& +e^{-2 G}\left[\frac{\partial^{2} \phi}{\partial r^{2}}+\left(\frac{(D-2}{r}+\frac{\partial}{\partial r}(F-G)\right) \frac{\partial \phi}{\partial r}\right] .
\end{aligned}
$$

If we put $D=4$, then (61) reduce to four dimensional d'Alembert operator as (33).

Finally, the $\{r r\}$ component of (60) can be written down as

$$
\frac{\partial}{\partial r}(F+G)=\frac{8 \pi r}{D-2}\left(\frac{\partial \phi}{\partial r}\right)^{2} .
$$

If we put $D=4$, then (63) reduce to (34).

The $\{i j\}$ component of (60) can be written as $\partial_{1}(F-G)-\frac{1}{r}\left[e^{2 G} \Lambda(D-2)-(D-3)\right]=$

$$
-\frac{16 \pi r e^{2 G}}{(D-2)} V(\phi)
$$

where $\hat{R}_{i j}(\hat{\sigma})=\Lambda(D-2) \hat{\sigma}_{i j}$. If we put $D=4$, then (64) reduce to (35).

\section{CONCLUSION}

In this present work, we study the EinsteinKlein-Gordon system in higher dimensional. We give the formulation of Ricci tensor, scalar curvature, and Einstein equation. If we put $D=4$, our formulations reduce to the four dimensional Einstein-KleinGordon system.

\section{ACKNOWLEDGMENT}

The work of this research is supported by Riset KK ITB 2021, P2MI FMIPA ITB 2021, and RistekBRIN 2021.

\section{REFERENCES}

[1] Akbar F T, Gunara B E, Iqbal M, and Susanto $\mathrm{H}$. Local and global existence of solutions to scalar equations on spatially flat universe as a background with non-minimal coupling. Adv. Theor. Math. Phys., 23 (1), 1-26, 2019. 
[2] Andersson L and Moncrief V. On the global evolution problem in $3+1$ gravity. J. Geom. Phys, 2001.

[3] Chae D. Global existence of spherically symmetric solutions to the coupled Einstein and nonlinear Klein-Gordon system. Class. Quantum Gravity, 18, 4589, 2001.

[4] Choquet-Bruhat Y. Solutions C dequations hyperboliques non lineaire. C. R. Acad. Sci., Paris 272, 3868, 1968.

[5] Christodoulou D. The problem of a self-graviting scalar field. Commun. Math. Phys, 105, 33761, 1986.

[6] Christodoulou D and Klainerman S. The Global Non-Linear Stability of the Minkowski Space. (Princeton Mathematical Series vol 41) (Princeton, NJ: Princeton University Press). 1993.

[7] Chrusciel P T. (1992). On the uniqueness in the large of solutions to the Einstein equations (strong cosmic censorship) Mathematical Aspects of Classical Field Theory (Seattle, WA, 1991) (Providence, RI: American Mathematical Society), pp 23573.

[8] Friedrich $\mathrm{H}$. On the global existence and the asymptotic behavior of solutions to the Einstein Maxwell Yang-Mills equations. J. Diff. Geom, 34 275345, 1991.
[9] Friedrich $\mathrm{H}$ and Rendall A. The Cauchy problem for the Einstein equations Einsteins Field Equations and their Physical Interpretation ed B G Schmidt (Berlin: Springer), 2000.

[10]Grillakis M. Regularity and asymptotic behaviour of the wave equation with a critical nonlinearity. Ann. Math. 132, 485509, 1990.

[11] Iqbal M, Akbar F T, and Gunara B E. (2019). Local existence of scalar wave equation on the Robertson-Walker universe as a background with $\mathrm{k}=0$. Journal of Physics: Conference Series 1204 (2019): 012131.

[12] Malec E. Self-graviting nonlinear scalar fields. J. Math. Phys. 38, 365067, 1997.

[13] Rendall A D. Local and global existence theorems for the Einstein equations Living Reviews in Relativity. (Rendall A D 2000 Preprint gr-qc/0001008), 2000.

[14] Rein G and Rendall A D. Global existence of solutions of the spherically symmteric Vlasov Einstein system with small initial data. Commun. Math. Phys. 150, 56183, 1992.

[15] Struwe M. Globally regular solutions to the u5 KleinGordon equation. Ann. Scuola Norm. Pisa, 15, 495513, 1988.

[16] Wijayanto M P, Akbar F T, Gunara B E. Local Existence of Classical Solutions to Scalar Field Equation on Spatially Compact Spacetime as a Background. Journal of Physics: Conference Series, 1949, 2021. 